\title{
Looking for the GAP effect in manual responses and the role of contextual influences in reaction time experiments
}

A.J.P. Faria Jr. and W. Machado-Pinheiro
Laboratório de Neurofisiologia do Comportamento, Departamento de Fisiologia e

Farmacologia, Instituto Biomédico, Universidade Federal Fluminense, Niterói, RJ, Brasil

\section{Correspondence \\ W. Machado-Pinheiro \\ Laboratório de Neurofisiologia do \\ Comportamento \\ Departamento de Fisiologia e \\ Farmacologia \\ Instituto Biomédico, UFF \\ Rua Hernani Mello, 101 \\ 24210-130 Niterói, RJ \\ Brasil \\ Fax: +55-21-2629-2414 \\ E-mail: walter@vm.uff.br}

Research supported by CAPES, CNPq and PROPP-UFF. A.J.P. Faria Jr. was the recipient of a CAPES fellowhip.

Presented at the XVIII Annual Meeting of the Federação de Sociedades de Biologia Experimental, Curitiba, PR, Brazil, August 27-30, 2003.

Received June 2, 2003 Accepted May 11, 2004

\begin{abstract}
When the offset of a visual stimulus (GAP condition) precedes the onset of a target, saccadic reaction times are reduced in relation to the condition with no offset (overlap condition) - the GAP effect. However, the existence of the GAP effect for manual responses is still controversial. In two experiments using both simple (Experiment $1, \mathrm{~N}=18$ ) and choice key-press procedures (Experiment 2, $\mathrm{N}=$ 12), we looked for the GAP effect in manual responses and investigated possible contextual influences on it. Participants were asked to respond to the imperative stimulus that would occur under different experimental contexts, created by varying the array of warning-stimulus intervals $(0,300$ and $1000 \mathrm{~ms})$ and conditions (GAP and overlap): i) intervals and conditions were randomized throughout the experiment; ii) conditions were run in different blocks and intervals were randomized; iii) intervals were run in different blocks and conditions were randomized. Our data showed that no GAP effect was obtained for any manipulation. The predictability of stimulus occurrence produced the strongest influence on response latencies. In Experiment 1, simple manual responses were shorter when the intervals were blocked $(247 \mathrm{~ms}, \mathrm{P}<0.001)$ in relation to the other two contexts ( 274 and $279 \mathrm{~ms}$ ). Despite the use of choice key-press procedures, Experiment 2 produced a similar pattern of results. A discussion addressing the critical conditions to obtain the GAP effect for distinct motor responses is presented. In short, our data stress the relevance of the temporal allocation of attention for behavioral performance.
\end{abstract}

Key words

- Reaction times

- Attention

- Preparation

- Expectancy

- Contextual influences

.............................

\section{Introduction}

There is general agreement that reaction times are reduced when a warning signal (WS) precedes the onset of a subsequent stimulus (imperative stimulus or IS) to which a response must be given (e.g., 1). Nevertheless, other studies have shown that the facilitation induced by the WS can be modulated by manipulations affecting different aspects of the experimental organization. One such manipulation includes, for example, the kind 
of WS employed - visual onset, visual offset or auditory warning $(2,3)$. In this respect, the offset of a visual stimulus has been handled in order to generate two distinct conditions, which are known to differently influence saccadic reaction times: the GAP and the overlap (OVR) conditions. The former is defined as the offset of a fixation point (FP) some milliseconds before the occurrence of an IS. In contrast, the OVR condition is defined as the maintenance of the FP until the end of a trial. The reduction observed in saccadic reaction times when the GAP condition is compared with the OVR condition is called the "GAP effect" (e.g., 4). Competing theories have been proposed to explain it (47 ), but there is as yet no agreement about the cause of such effect. Moreover, some groups claim that there is a GAP effect for manual reaction times (MRTs) $(7,8)$, while others do not agree $(5,9)$.

A second critical example of experimental manipulation is related to the duration and the organization of the intervals between WS and IS (10-12). In fact, the interval between the WS and the IS, known as the foreperiod, plays a central role in manual reaction time paradigms since it represents the period of time during which the preparatory phases of reaction processes primarily take place (13). The foreperiod provides a temporal frame of reference for IS occurrence. Changes in the duration and variability of the WS-IS intervals have important effects on the responsiveness of subjects. Therefore, time uncertainty created by the manipulation of intervals is a major factor affecting the level of motor preparation and, consequently, response latency (10-14).

Actually, at times, subtle modifications in the experimental design are able to completely modify results and prevent usual comparisons, a fact which complicates the interpretation of data obtained by different groups. In this respect, another aspect in the experimental design that has not received the proper attention is related to the context in which the tasks are inserted. Examples of such contextual influences are found in experiments that employ a "blocked" (maintaining conditions or variables constant within a block of trials) or "non-blocked" (randomizing conditions or variables within a block of trials) organization. We have recently found that visual onset and visual offset used as WS produced different effects on manual latencies, and more important, those influences were modulated by the experimental context (blocked $v s$ non-blocked) adopted (15). Since the visual offset represents the critical condition for any study related to the GAP effect, our results suggest that the facilitation induced by the visual offset - the GAP effect, could also be modulated by changes in the experimental context. Therefore, we are inclined to consider that at least a portion of the aforementioned disagreement in relation to the GAP effect might be related to differences in such experimental context used by different groups, which were not usually considered.

The aim of this study is to discuss the effects that manipulations of the experimental context have on MRTs, employing both GAP and OVR conditions. By means of two experiments, we will investigate the occurrence of the GAP effect for manual responses and search for possible contextual influences on it. We expect to show the importance of considering contextual influences on manual responses and contribute to the understanding of such influences on behavioral performance.

\section{Experiment 1}

In this first experiment, we determined the existence of the GAP effect for manual responses using the simple reaction time paradigm. In order to test for a putative influence of contextual procedures, GAP and OVR trials were inserted into three different experimental contexts according to the array of conditions and intervals adopted. 
Methods

Participants. Nine male and nine female undergraduate or graduate students of Universidade Federal Fluminense participated voluntarily in the experiment. They were all between 20 to 30 years old, had normal or corrected vision and were right-handed as defined by the Edinburgh Inventory (16) They were all naive with respect to the goals of the present experiment. The local Ethics Committee approved the procedures adopted and written informed consent was obtained from the participants prior to the study.

Apparatus. The participants were tested in a sound-attenuated room under dim ambient light. They sat in front of a 14-inch video monitor $\left(0.2 \mathrm{~cd} / \mathrm{m}^{2}\right.$ luminance $)$, with their head positioned on a chin-and-front rest and with their eyes positioned at approximately $57 \mathrm{~cm}$ from the screen. The timing of the presentation of auditory and visual stimuli and the recording of key pressings were performed by a PC-486 microcomputer running customized software written in MEL2 language (Psychology Software Tools, Pittsburgh, PA, USA).

Procedure. Each trial began with the presentation of an FP at the center of the screen. Seven hundred milliseconds later $(700 \pm 50 \mathrm{~ms})$, a $2000-\mathrm{Hz}, 67-\mathrm{dB}, 50-\mathrm{ms}$ tone ("beep" - WS) was emitted from a loudspeaker placed directly below the screen. After the appearance of the FP, two possible events were expected according to the GAP or OVR conditions. In the GAP condition, simultaneous to WS presentation, the FP went off. In the OVR condition, the FP remained on until the end of the trial. At a variable interval following the WS (which occurred in both conditions), a white filled circle (IS), with an $11.5 \mathrm{~cd} / \mathrm{m}^{2}$ luminance, appeared on the screen $8^{\circ}$ to the right or left of the FP. Participants were told to ignore changes in the FP and to use the "beep" as a preparatory signal for the occurrence of the IS, pressing a key with their right index finger as soon as they detected it.

The intervals between WS and the onset of IS were $0,300(300 \pm 50 \mathrm{~ms})$ and $1000 \mathrm{~ms}$ $(1000 \pm 100 \mathrm{~ms})$ which had the same probability of occurrence. After each manual response, its latency, in milliseconds (MRTs), appeared on the screen for $1000 \mathrm{~ms}$. The next trial began immediately after this presentation.

Participants were required to perform a series of four sessions on separate days (approximately $30 \mathrm{~min}$ per session). Each session consisted of 288 trials, divided into 6 blocks of 48 trials with one or 2 min of rest between each block. The first session was only for training, and the data were not considered. Any MRT shorter than $100 \mathrm{~ms}$ or longer than $700 \mathrm{~ms}$ was considered to be an error and generated a written message with the words "anticipation" or "slow response", respectively, which remained on the screen for $1000 \mathrm{~ms}$. These error trials were repeated at the end of each session.

There were three possible experimental contexts. The first was the "non-blocked" context (NBL), where condition (GAP and OVR) and interval (0, 300 and $1000 \mathrm{~ms})$ were randomized throughout the 6 blocks of trials. The second was the "blocked condition" (BLGO), in which condition was run in different blocks of trials, but interval was randomized. The third context was the "blocked interval" (BLINT), in which the intervals were run in different blocks of trials, but condition was randomized. It is important to stress that the participants were not explicitly informed about the differences in contexts, conditions or intervals. The variables were organized in such a way that all of them were run equally throughout the experiment.

The median MRTs obtained on the second, third and fourth days were submitted to analysis of variance (ANOVA) in which "context" (NBL, BLGO and BLINT), "condition" (GAP and OVR) and "interval" ( 0 , 300 and $1000 \mathrm{~ms}$ ) were used as within- 
subject factors. When necessary, we also performed a post hoc analysis using the Newman-Keuls method. The level of significance was set at $\mathrm{P}<0.05$ in all analyses.

\section{Results and Discussion}

The error trials corresponded to $4.17 \%$ (648 errors in 15,552 trials), and most of them were anticipations (619 anticipations and 29 slow responses). The errors were also equally distributed among contexts, conditions and intervals.

ANOVA showed a significant effect of context $(\mathrm{F}(2,34)=23.866, \mathrm{P}<0.001)$ and of interval $(\mathrm{F}(2,34)=22.190, \mathrm{P}<0.001)$ but not of condition $(\mathrm{F}(1,17)=1.301, \mathrm{P}=$ 0.269). Regarding context, BLINT (247 ms) was significantly different from the others $(\mathrm{P}$ $<0.001)$, i.e., BLGO (274 ms) and NBL (279 $\mathrm{ms}$ ), which did not differ from each other. Regarding interval, MRTs for the 0 -ms interval $(285 \mathrm{~ms})$ were longer $(\mathrm{P}<0.001)$ than those obtained for the $300-\mathrm{ms}(261 \mathrm{~ms})$ and 1000-ms (254 ms) intervals, which, however did not differ from each other. More-

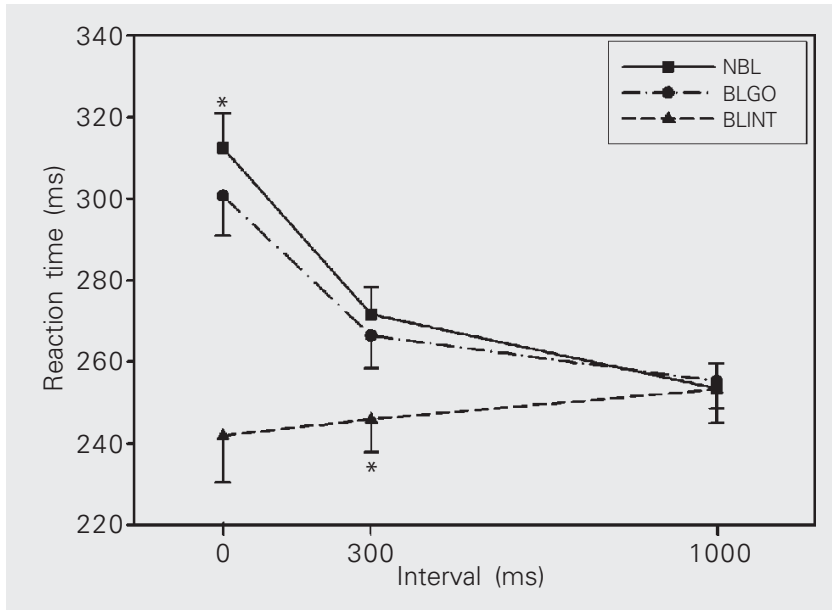

Figure 1. Mean latency of manual reaction times (ms) obtained in the first experiment as a function of interval (ms) and experimental context (NBL, BLGO and BLINT). The error bars indicate \pm SEM. NBL $=$ nonblocked context; $\mathrm{BLGO}=$ blocked condition; $\mathrm{BLINT}=$ blocked interval. Post hoc analysis showed that the three contexts were different at 0 ms interval, whereas at $300 \mathrm{~ms}$ only the BLINT context was different. No difference was observed at $1000 \mathrm{~ms}\left({ }^{*} \mathrm{P}<0.05\right.$ for all comparisons). Number of participants $=18$ in each group. over, all interactions were significant: between context and condition $(F(2,34)=6.015$, $\mathrm{P}=0.005)$, between context and interval $(\mathrm{F}(4,68)=44.767, \mathrm{P}<0.001)$, between condition and interval $(\mathrm{F}(2,34)=4.251, \mathrm{P}=$ $0.022)$, as well as the triple interaction among context, condition and interval $(\mathrm{F}(4,68)=$ $2.858, \mathrm{P}=0.03)$. The main results of these interactions are presented below.

Figure 1 shows the results of the interaction between context and interval. Post hoc analysis showed that within the NBL and BLGO contexts, there was a difference among the three intervals $(\mathrm{P}<0.008$ for all comparisons). Nonetheless, for the BLINT context the only difference occurred between the 0 and 1000-ms intervals $(P=0.016)$. More importantly, all contexts differed at the 0 -ms interval $(312,301$ and $242 \mathrm{~ms}$, respectively, for NBL, BLGO and BLINT; $\mathrm{P}<0.005$ for all), but not at the 300-ms interval $(271,266$ and $246 \mathrm{~ms}$, respectively) when only the BLINT context differed $(\mathrm{P}<0.001)$. No differences were found at the 1000-ms interval (253, 255 and $253 \mathrm{~ms}$, respectively).

These results clearly show the effects of contextual manipulations on MRTs. As we can see, the BLINT context was the one that generated the shortest MRTs. This facilitation was expected due to the high temporal predictability inherent to this context: participants were able to use the blocked array of intervals in order to "precisely" predict the moment of target occurrence. To reinforce this interpretation, NBL context, the most unpredictable one, produced the longest MRTs.

The experiment summarized in Figure 2 was designed to verify the contextual influences on GAP/OVR paradigms (interaction among context, condition and interval). Figure $2 \mathrm{~A}$ shows the results obtained for the NBL context: differences between GAP and OVR were only found at the $300-\mathrm{ms}$ interval (277 and $266 \mathrm{~ms}$, respectively, $\mathrm{P}=0.002$ ). For the BLGO context (Figure 2B), GAP and OVR were different at the 0 -ms interval (305 and $297 \mathrm{~ms}$, respectively, $\mathrm{P}=0.008$ ) and 
$1000-\mathrm{ms}$ interval $(260$ and $251 \mathrm{~ms}$, respectively, $\mathrm{P}=0.018$ ). Finally, for the BLINT context (Figure 2C), the most predictable one, no differences were found between GAP and OVR. Therefore, for all contexts and intervals, when simple MRT was used, no facilitation for the GAP condition (the "GAP effect") was obtained.

Thus, with the experimental procedures employed in the first experiment it was not possible to find any GAP effect for manual responses. Bekkering's group proposed in two different papers $(7,8)$ that the GAP effect may be specific for spatially oriented responses. On this basis, the procedures used in the first experiment would not be able to evidence any GAP effect due to the use of simple (not spatially oriented) manual response. In their papers $(7,8)$, Bekkering, Pratt and co-workers found a significant GAP effect when participants had to make a saccade to the target location (GAP effect ranging from 42 to $67 \mathrm{~ms}$ ), to move a handle to the left or to the right (GAP effect ranging from 24 to $50 \mathrm{~ms}$ ), and also when a choice key-press response was used (significant GAP effect ranging from 6.3 to $11 \mathrm{~ms}$ ). However, when simple MRT was used, manual responses were $4 \mathrm{~ms}$ faster in the
OVR than in the GAP condition, i.e., no GAP effect was found (7).

Therefore, according to the premise of Bekkering, Pratt and co-workers, the absence of the GAP effect observed in Experiment 1 should be simply related to the use of a non-spatial key-press procedure. In order to investigate this premise, we decided to run a second experiment using choice key-press responses.

\section{Experiment 2}

In this experiment a choice MRT procedure was used in order to determine if, in fact, the use of a spatially oriented response is an essential condition in order to generate a significant GAP effect for manual responses.

\section{Methods}

Participants. Six male and six female undergraduate or graduate students of Universidade Federal Fluminense participated voluntarily in the experiment. They were all 20 to 36 years old, had normal or corrected vision and were right-handed as defined by the Edinburgh Inventory (16). They were all
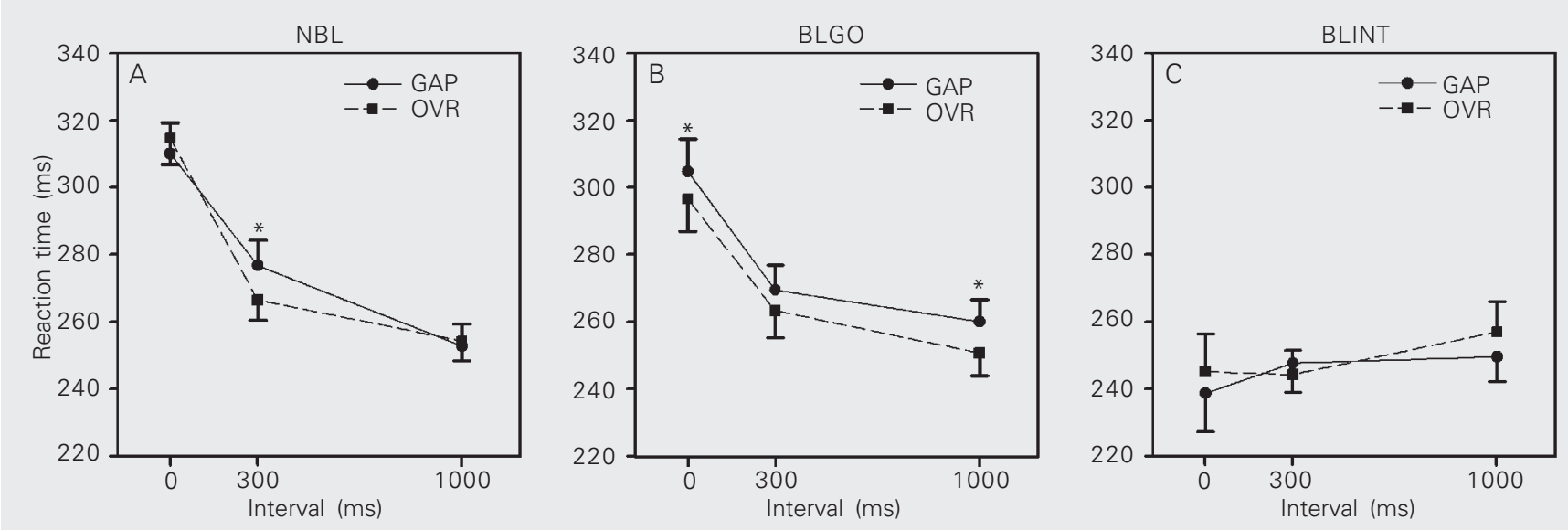

Figure 2. Mean latency of manual reaction times (ms) obtained in the first experiment as a function of interval (ms), condition (GAP and overlap, OVR) and the three experimental contexts: $A, N B L ; B, B L G O$ and $C, B L I N T$. The error bars indicate \pm SEM. For abbreviations, see legend to Figure 1. Post hoc analysis showed that for the NBL context (A), difference between GAP and OVR conditions was found at 300-ms interval. For the BLGO context (B), GAP and OVR conditions differed at 0 and $1000 \mathrm{~ms}$. No difference was found for the BLINT context (C). ${ }^{*} \mathrm{P}<0.05$ for all comparisons. Number of participants $=18$ in each group. 
naive with respect to the goals of the present experiment. The local Ethics Committee approved the procedures adopted and written informed consent was obtained from the participants prior to the study.

Apparatus. The apparatus used in this second experiment was exactly the same as that used in Experiment 1.

Procedure. The procedure used in this second experiment was the same as that used in the previous experiment, except for two differences. The first, as mentioned, was the introduction of the choice key-press procedure, a spatially oriented response: participants had to press a key with the left or right index finger according to the side of IS occurrence - a compatible stimulus-response mapping. The second was the use of only one experimental session, which lasted about 40 min, with 432 trials, 144 for each context. The NBL context was divided into 2 blocks of 72 trials; BLGO was also divided into two blocks of 72 trials, one for each condition (GAP and OVR), and BLINT was divided into three blocks of 48 trials, one for each interval $(0,300$ and $1000 \mathrm{~ms})$. All variables were counterbalanced across participants, just as in Experiment 1.

The median MRT values were submitted to ANOVA in which "hand" (left and right), "context" (NBL, BLGO and BLINT), "condition" (GAP and OVR) and "interval" (0, 300 and $1000 \mathrm{~ms}$ ) were used as withinsubject factors. When necessary, we also performed a post hoc analysis using the Newman-Keuls method. The level of significance was set at $\mathrm{P}<0.05$ in all analyses.

\section{Results and Discussion}

As expected, the mean manual latencies in this second experiment increased to 293 ms against the 267 ms obtained in Experiment 1 , due to the use of the choice keypress procedure. The error trials corresponded to only $1.75 \%$ (91 errors in 5184 trials) and they were almost equally distributed among hands, contexts, conditions, and intervals.

ANOVA showed that context $(\mathrm{F}(2,22)=$ 9.985, $\mathrm{P}<0.001)$, interval $(\mathrm{F}(2,22)=29.371$, $\mathrm{P}<0.001)$ and the interaction between them $(\mathrm{F}(4,44)=22.390, \mathrm{P}<0.001)$ were significant sources of variance. There was no main effect of hand (left or right) or any interaction with any other variable $(\mathrm{P}>0.05$ for all). Figure 3 shows the main results obtained. Due to the absence of any significance or interaction, data for both hands were pooled. Note that, despite the use of a choice key-
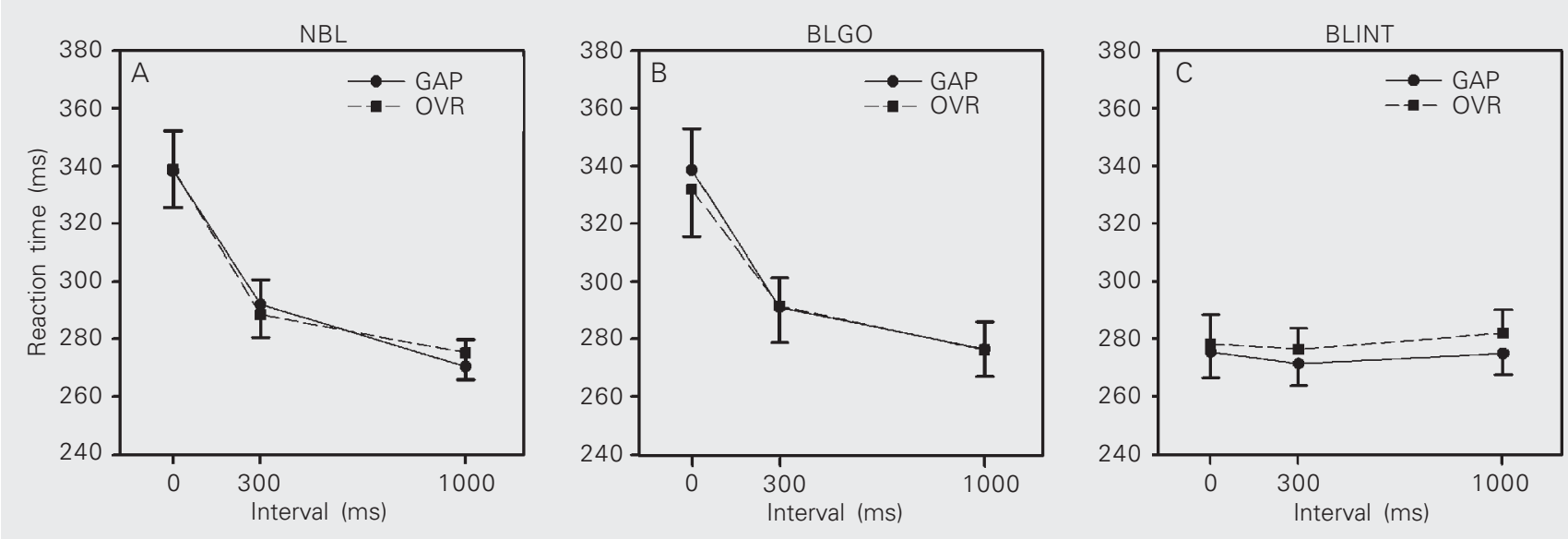

Figure 3. Mean latency of manual reaction times (ms) obtained in the second experiment as a function of interval (ms), condition (GAP and overlap, OVR) and the three experimental contexts: $A, N B L ; B, B L G O$ and $C, B L I N T$. The error bars indicate \pm SEM. For abbreviations, see legend to Figure 1. No difference between GAP and OVR conditions was found ( $P>0.05$ for all comparisons). Number of participants $=12$ in each group. 
press paradigm, no significant difference between GAP and OVR conditions was obtained for any experimental context, i.e., there was no evidence of the GAP effect.

Therefore, our data do not support the premise pointed out by Bekkering and coworkers $(7,8)$ : using either choice (spatially oriented) or simple (not spatially oriented) manual responses we could not evidence any significant GAP effect. Taken together, our results agree with those obtained by ReuterLorenz et al. (5) who did not found a significant GAP effect using also choice key-press responses.

\section{General Discussion}

On the basis of their data, both ReuterLorenz et al. $(5,6)$ and Kingstone and Klein (9) proposed the so-called "two-component model" to explain the GAP effect. According to these investigators, the reduction in motor responses observed in the GAP condition should have two components: i) a warning component which follows any warning event and is common to both saccadic and manual responses, and ii) a fixation offset component which is specific for saccades and is observed when the FP is turned off. The latter component may be mediated by the fixation neurons located in the rostral pole of the superior colliculus. According to this view, the offset of a foveal stimulus would decrease the inhibitory activity of fixation neurons on collicular neurons involved in eye movements, decreasing saccade latency (e.g., 5,6,9). Therefore, as proposed by Kingstone and Klein (9) and Reuter-Lorenz et al. (5), the WS (beep) was used in our procedures in both GAP and OVR paradigms as a control for the first component, the nonspecific one. Since we were not able to evidence any facilitation in the GAP condition, despite the use of simple or choice manual responses, our data support those that claim that no evidence of facilitation for the GAP paradigm can be evidenced for manual responses when the nonspecific warning component of the GAP effect is controlled. According to this view, using such control, the GAP effect can be found only for saccadic reaction times due to the fixation offset component $(5,6,9)$.

However, since Bekkering, Pratt and coworkers have systematically found a significant GAP effect for manual responses, we propose that other factors must be considered in this discussion. The first one is related to the use of mutually exclusive motor responses. For example, in the studies by Bekkering, Pratt and co-workers, when pointing movements were used (to move a handle to the left or to the right) a reliable and significant GAP effect was obtained - 24 to $50 \mathrm{~ms}(7,8)$. However, when the choice keypress was introduced, the magnitude of the GAP effect was considerably smaller -6.3 to $11 \mathrm{~ms}$ (7), or did not reach significance, as reported by Reuter-Lorenz et al. (5). Nevertheless, to move a handle is a mutually exclusive movement, i.e., you cannot move it to both sides at the same time, while it is perfectly possible to press both left and right keys simultaneously. Thus, one possibility is that the use of mutually exclusive motor responses could be a determinant factor to generate a reliable GAP effect. The second point is related to the use of proximal $v s$ distal limb muscles. Note that in key-press procedures distal muscles are employed, while more proximal ones are used when moving a handle is required. There are some lines of evidence supporting a role for the superior colliculus in guiding the arm during reach movements via the control of proximal limb musculature (17-20). Strong correlations between electromyographic activity of arm muscles and neuronal activity of a collicular "reach cell" have been found for proximal muscles. However, the correlation obtained for distal muscles, such as the flexor digitorum superficialis, was much less frequent and weak (18), confirming previous findings linking the superior colliculus to orienting movements of the eyes, head and arms (e.g., 
17,21). So, if collicular mechanisms are, in fact, mediating the facilitation in the GAP paradigm, the GAP effect for key-press responses should be minimal or even nonexistent. Obviously, these two considerations deserve further analysis in futures experiments.

As mentioned earlier, the objective of the present study was to discuss the influence of the experimental context on the occurrence and magnitude of the GAP effect for manual responses. However, in two different experiments we were not able to evidence any signal of the GAP effect. In fact, the main findings of our study are related to the influence of IS predictability on motor latencies in general. By changing the context of trial presentation, it was possible to modulate the influence of the WS on MRTs to visual stimuli. As shown in Figure 1, no differences between the three contexts were found for the 1000-ms interval. We believe that, with this long interval, endogenous top-down influences owing to the prediction of, or total certainty about IS occurrence induced such a high level of motor preparation/expectancy as to overcome any effect related to differences in experimental context, differences which, in our view, are more subtle. This interpretation is corroborated by the enhanced influence of contextual effects observed at the 0 -ms interval, when all contexts were different: at this short interval, the above mentioned top-down influence would be minimal and so contextual influences have more chance to appear. At $300 \mathrm{~ms}$, an intermediate interval, when probably contextual effects and top-down influences are coexisting more or less equally, differences among contexts, although smaller ones, can still be found.

We also found that the BLINT context was the one where top-down influences were more evident. In this context, MRTs for the three intervals were almost the same, with only a small difference $(11 \mathrm{~ms})$ between the 0 - and 1000-ms intervals. The greater decrease in motor latencies obtained at the 0 ms interval compared to the other two contexts showed us just how important the IS predictability is. When participants are able to predict IS occurrence with a high certainty, they can prepare themselves even before the WS, using cues other than the WS (for example, the end of the previous trial) in order to anticipate IS onset. This happens primarily at extremely short intervals, which do not allow suitable motor preparation during the foreperiod (13). This effect is so clear that participants are able to be as fast (or even faster) for these short intervals as for the long ones when a high level of preparation is achieved, before the foreperiod.

Such top-down influence has been proposed by some investigators (22-26). In our view, it is related to the motor preparation that follows the WS, and is strongly dependent on precise timing of IS occurrence, i.e., predictability. In reaction time experiments, this motor-preparation activity occurs during the foreperiod, or even before, as in the case of the 0 -ms interval and contributes to shortening of motor latencies. In fact, using experimental procedures that involve an instructed delay period between a cue and the triggered movement, some studies have documented such motor-preparation activity related to various motor centers: the motor cortex (27), the basal ganglia (28), the spinal interneurons (29) and the superior colliculus (30).

All the interpretation for the temporal context effects obtained in this paper was based on the classical modal or strategic view that has been constructed since the studies by Klemmer (10), Bertelson (11) and Drazin (12), for example, and that was reviewed by Niemi and Näätänem (13). The strategic view is based on the preparatory state of the participants and their intentional ability to anticipate the moment of IS occurrence (temporal expectancy). However, a more recent view of these effects, the conditioning view, was proposed by Los and coworkers $(31,32)$. According to this new 
approach, the preparatory processes involved in anticipating IS occurrence would correspond to a conditioned response unintentionally elicited by WS. The conditioning view highlights the similarity between the design used in our experimental approach and those used to examine trace conditioning in animals. Accordingly, the IS may serve as an unconditioned stimulus which entails a tendency to respond as an unconditioned response due to the instructions of the task. The WS would serve as a conditioned stimulus, and so, after some practice, a conditioned response - in this case a tendency to respond - would develop. In short, the nonspecific preparation acquired unintentionally would be reinforced if participants' expectancy coincided with IS occurrence, leading to a reduction in MRT, and would be extinct if the IS occurred before participants' expectancy. Thus, when the interval between WS and IS is maintained constant in a block, such as in our BLINT context, manual latencies would be smaller, due to the reinforcement of the conditioned response - a premise that was completely confirmed by our results. Despite the growing interest in the conditioning view, a discussion and comparison between the strategic and conditioning views was not a goal of this study, nor were our procedures designed to address it.

This discussion about predictability (tem- poral expectancy) and contextual manipulations clearly stresses the importance of both in the determination of reaction times. In fact, the temporal allocation of attention has recently received the proper recognition (2326) as a major factor modulating reaction times. Our data also indicate that the search for contextual effects on reaction time experiments must be focused on short intervals, where endogenous top-down influences are minimal. In conclusion, ignoring the contextual effects might lead to misinterpretation of experimental data and generate controversies in the literature. The results presented here and others obtained by our group (33, and Machado-Pinheiro W, Faria Jr. AJP, Gawryszewski LG, Ranvaud R and Ribeiro-do-Valle LE, unpublished results) stress the importance of contextual manipulations, primarily those related to motorpreparation and top-down influences on MRTs.

\section{Acknowledgments}

We would like to thank Dr. Ronald Ranvaud and Dr. Marcus Vinícius C. Baldo, from Instituto de Ciências Biomédicas, Universidade de São Paulo, for their valuable comments on an early version of the manuscript.

\section{References}

1. James W (1890/1950). Attention. In: The Principles of Psychology. Vol. 1. Dover Publications, New York, 402-458.

2. Ross SM \& Ross LE (1981). Saccade latency and warning signals: effects of auditory and visual stimulus onset and offset. Perception and Psychophysics, 29: 429-437.

3. Pratt J \& MacAuliffe J (2001). The effects of onsets and offsets on visual attention. Psychological Research, 65: 185-191.

4. Fischer B \& Weber H (1993). Express saccades and visual attention. Behavioral and Brain Sciences, 16: 553-610.

5. Reuter-Lorenz PA, Hughes HC \& Fendrich R (1991). The reduction of saccade latency by prior offset of the fixation point: An analysis of the GAP effect. Perception and Psychophysics, 49: 167-175.

6. Reuter-Lorenz PA, Oonk HM, Barnes LL \& Hughes HC (1995). Effects of warning signals and fixation point offsets on the latency of pro-versus antisaccades: implications for an interpretation of the GAP effect. Experimental Brain Research, 103: 287-293.

7. Bekkering H, Pratt J \& Abrams RA (1996). The GAP effect for eye and hand movement. Perception and Psychophysics, 58: 628635.

8. Pratt J, Bekkering H, Richard AA \& Adam J (1999). The GAP effect for spatially oriented responses. Acta Psychologica, 120: 1-12.

9. Kingstone A \& Klein RM (1993). Visual offsets facilitate saccadic latency: does predisengagement of visuospatial attention mediate the GAP effect? Journal of Experimental Psychology: Human Perception and Performance, 19: 1251-1265.

10. Klemmer ET (1956). Time uncertainty in simple reaction time. Journal of Experimental Psychology, 51: 179-184.

11. Bertelson P (1967). The time course of preparation. Quarterly 
Journal of Experimental Psychology, 19: 272-279.

12. Drazin DH (1961). Effects of foreperiod, foreperiod variability, and probability of stimulus occurrence on simple reaction time. Journal of Experimental Psychology, 62: 43-50.

13. Niemi $P$ \& Näätänem R (1981). Foreperiod and simple reaction time. Psychological Bulletin, 89: 133-162.

14. Weber H, Biscaldi M \& Fischer B (1995). Intertrial effects of randomization on saccadic reaction times in human observers. Vision Research, 35: 2615-2642.

15. Machado-Pinheiro W, Faria Jr AJP, Gawryszewski LG \& Ribeiro-doValle LE (2004). Experimental context modulates warning signal effects. Brazilian Journal of Medical and Biological Research (in press).

16. Oldfield RC (1971). The assessment and analysis of handedness: The Edinburgh Inventory. Neuropsychologia, 9: 97-113.

17. Stuphorn V, Bauswein E \& Hoffman K-P (1996). Cortico-collicular control of arm movements. In: Lacquaniti F \& Viviani P (Editors), Neural Bases of Motor Behaviour. Kluwer Academic Publishers, Dordrecht, Netherlands, 185-204.

18. Stuphorn V, Hoffman K-P \& Miller LE (1999). Correlation of primate superior colliculus and reticular formation discharge with proximal limb muscle activity. Journal of Neurophysiology, 81: 1978-1982.

19. Werner W, Dannenberg S \& Hoffman KP (1997). Arm-movementrelated neurons in the primate superior colliculus and underlying formation: comparison of neuronal activity with EMGs of muscles of the shoulder, arm and trunk during reaching. Experimental Brain Research, 115: 191-205.

20. Werner W, Hoffman KP \& Dannenberg S (1997). Anatomical distribution of arm-movement-related neurons in the primate superior colliculus and underlying reticular formation in the comparison with visual and saccadic cells. Experimental Brain Research, 115: 206216.

21. Stuphorn V, Bauswein E \& Hoffman KP (2000). Neurons in the primate superior colliculus coding for arm movements in gazerelated coordinates. Journal of Neurophysiology, 83: 1283-1299.

22. Trappenberg TP, Dorris MC \& Klein RM (2001). A model of saccade initiation based on the competitive integration of exogenous and endogenous signals in the superior colliculus. Journal of Cognitive Neuroscience, 13: 256-271.

23. Coull JT, Frith CD, Büchel C \& Nobre AC (2000). Orienting attention in time: behavioral and neuroanatomical distinction between exogenous and endogenous shifts. Neuropsychologia, 38: 808819.

24. Nobre AC (2001). Orienting attention to instants in time. Neuropsychologia, 39: 1317-1328.

25. Nobre AC, Coull JT, Frith CD \& Mesulam MM (1999). Orbitofrontal cortex is activated during breaches of expectation in tasks of visual attention. Nature Neuroscience, 2: 11-12.

26. Coull JT \& Nobre AC (1998). Where and when to pay attention: the neural systems for directing attention to spatial locations and to time intervals as revealed by both PET and fMRI. Journal of Neuroscience, 18: 7426-7435.

27. Tanji J \& Evarts EV (1976). Anticipatory activity of motor cortex neurons in relation to direction of an intended movement. Journal of Neurophysiology, 39: 1062-1068.

28. Alexander GE (1987). Selective neuronal discharge in monkey putamen reflects intended or planned limb movements. Experimental Brain Research, 67: 623-634.

29. Prut $Y$ \& Fetz EE (1999). Primate spinal interneurons show premovement instructed delay activity. Nature, 401: 590-594.

30. Munoz DP \& Wurtz RH (1995). Saccade related activity in the monkey superior colliculus. II. Spread of activity during saccades. Journal of Neurophysiology, 73: 2334-2348.

31. Los SA, Knol DL \& Boers RM (2001). The foreperiod effect revisited: conditioning as a basis for nonspecific preparation. Acta Psychologica, 106: 121-145.

32. Los SA \& Van Den Heuvel CE (2001). Intentional and unintentional contributions to nonspecific preparation during reaction time foreperiods. Journal of Experimental Psychology: Human Perception and Performance, 27: 370-386.

33. Machado-Pinheiro W, Gawryszewski LG \& Pereira Jr A (2003). Manual responses to visual stimuli: early and late facilitatory effects due to the offset of a peripheral cue. Arquivos Brasileiros de Oftalmologia, 66 (Suppl): 105-113. 